l'énergie et du flux d'ions contenus dans le plasma avec la puissance laser.

Les courbes obtenues montrent qu'en agissant sur l'énergie du faisceau laser, il est possible de controler dans des conditions assez satisfaisantes les caractéristiques du plasma obtenu par ionisation d'une cible solide.

Il est donc possible de remplacer avantageusement en tant que préionisation, les canons à plasma par ce genre de source, en particulier lorsque la cible est un granule en suspension électrostatique.

Le support étant alors supprimé, on peut créer le plasma en un point de la structure et en un temps choisis volontairement sans avoir de perturbation due au support.
Signalons immédiatement que dans les régimes pulsés dans les dispositifs accélérateurs, le support ne gene pas énormément l'action du champ h.f. La bouffée de plasma créé constitue bien pour le champ h.f. intense une charge appropriée susceptible d'interargir avec ce dernier [3].

\section{Références}

1. P. Briand, T. Consoli, G. Mourier, L. Slama et P. Vial, Second European Conference, Stockholm, aoat 1967.

2. P. Vial, Bull. Inform. Sci. Tech. No. 116 (1967) 85.

3. P. Briand, P.Grelot, G. Mourier, L. Slama et P. Vial, Comptes Rend. (a paraitre Sept. 1967).

\title{
POWER BROADENING AND COLLISION BROADENING OF GAS LASER TRANSITIONS
}

\author{
P. T. BOLWIJN* and C. Th. J. ALKEMADE \\ Physics Laboratory, State University Utrecht, The Netherlands
}

Received 20 September 1967

\begin{abstract}
From measured tuning characteristics of the amplitude of laser power modulation in single-mode operation, power broadening as well as collision broadening of the laser transition is shown to be relatively important and can be studied separately. It is also found that the d.c. laser power is proportional to the square of the gain.
\end{abstract}

The effect of collisions on the single-mode d.c. power-tuning characteristics of a $\mathrm{He}-\mathrm{Ne}$ laser has been shown to be relatively important at typical operation conditions $[1,2]$. The linewidth parameters associated with "hard" and "soft" collision processes have been determined from these characteristics on the basis of Lamb's semi-classical third-order theory [3], modified to incorporate collision broadening of the laser transition. As Lamb's theory ignores power broadening [cf. 4] such a determination of the linewidth parameters is valid for low excitation levels only, in particular at not too high gas pressures $[2,5]$. In this letter we show from experiments the relative importance of power broadening. The effect of power broadening clearly shows up in the tuning characteristics of

* Now with Philips' Research Laboratories, Eindhoven, The Netherlands. the amplitude of the laser modulation obtained by resonator $-Q$ or excitation-density modulation [6-9], even at low excitation level (say, relative excitation $\eta$, as defined in ref. $3, \eta \geqslant 1.05$ ).

Let us compare three theoretical expressions for the d.c. laser-power output in single-mode operation. Smith [10] has derived an expression for the laser power travelling one way inside the resonator for arbitrary excitation level, ignoring the standing-wave nature of the radiation. His analysis is valid for arbitrary ratio $r<1$ of power-plus collision broadened width $\gamma_{\mathbf{h}}^{\prime}$ to Doppler width $\Delta \nu_{\mathrm{D}}$ of the atomic resonance line. If, for simplicity, $r \ll 1$ it holds for the d.c. laserpower output $w$

$$
w \propto\left\{\eta^{2} \exp \left[-2\left(\nu-\nu_{\mathrm{O}}\right)^{2} /\left(0.6 \Delta \nu_{\mathrm{D}}\right)^{2}\right]-1\right\},
$$

where $\nu$ is the laser oscillation frequency, which is approximately equal to the resonance frequency of the resonator and $\nu_{0}$ is the center frequency 


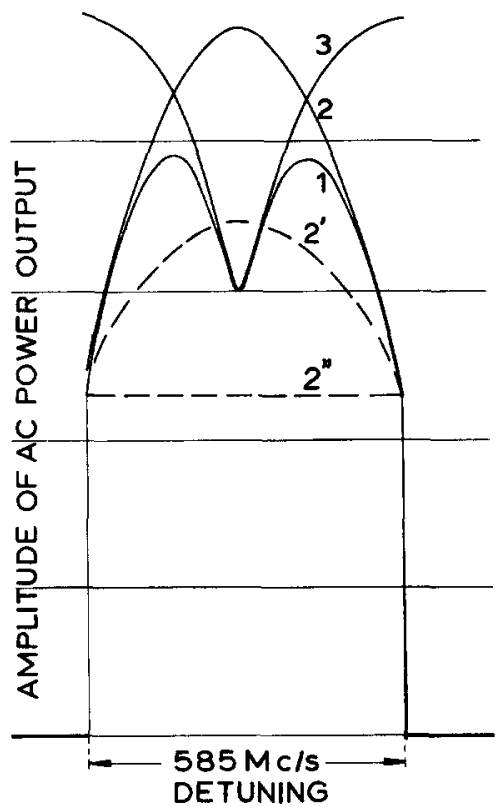

Fig. 1. Amplitude of laser-output power modulation versus resonator detuning of excitation-modulated laser. Curve 1 is experimental, and curves $2,2^{\prime}$ and $2^{n}$ are theoretical, disregarding the standing-wave nature of the radiation in the resonator. Curve 2 involves the square of the gain curve, whereas the curves 2 ' and $2^{n}$ involve the first power of the gain curve. Curve 3 describes the increased saturation around the line center as derived from curves 1 and 2 .

of the atomic transition, $\Delta \nu_{\mathrm{D}}$ is the full Doppler width at half-maximum of the gain curve, and $\eta$ is the relative excitation.

Disregarding the standing-wave nature of the radiation, Lamb's third-order theory yields, if $r \ll 1 *$

$$
w \propto\left\{\exp \left[-\left(\nu-\nu_{\mathrm{O}}\right)^{2} /\left(0.6 \Delta \nu_{\mathrm{D}}\right)^{2}\right]-\eta^{-1}\right\} .
$$

This may be compared with the expression found by, for instance, Tobias [11]

$$
\left.w \propto\left\{\eta \exp \left[-\left(\nu-\nu_{0}\right)^{2} / 0.6 \Delta \nu_{\mathrm{D}}\right)^{2}\right]-1\right\} .
$$

We note again that power broadening has been neglected in the derivation of eqs. (2) and (3).

From experimental tuning characteristics of the d.c. power output at large detuning it is difficult to differentiate between eqs. (1), (2) and (3). The a.c. power output arising from laser modulation techniques, however, is very sensitive to the differences predicted by these equations. As $\eta \propto N Q$ the theoretical laser power modulation as a function of resonator detuning $\approx\left(\nu-\nu_{0}\right)$ can easily be found for both excitation-density $(N)$ and resonator-quality $(Q)$ modulation.

Curve 1 in fig. 1 is an experimental curve of the amplitude of laser-output power modulation as a function of resonator detuning. (The pronounced dip near the line center should be distinguished from the Lamp-dip in the d.c. power characteristic). The modulation was obtained by a small modulation of the d.c. discharge current at $10 \mathrm{kc} / \mathrm{s}$. The laser used was a single-mode $1.15 \mu \mathrm{He}-20 \mathrm{Ne}$ laser [12], which was thermally tuned. The possible error in the frequency calibration of the detuning scale is $5-10 \%$ [13]. The total gas pressure was $1.9 \mathrm{Torr}$, and the $\mathrm{He}-20 \mathrm{Ne}$ ratio $5: 1$. The relative excitation $\eta$ is in fig. 1 $\eta \approx 1.4$ [cf. 8].

Curve 2 is the corresponding theoretical curve derived from eq. (1), whereas the curves 2 ' and $2^{\prime \prime}$ were derived from eqs. (3) and (2), respectively $¥$. These curves were fitted to curve 1 at threshold detuning. It should be recalled that the standing-wave nature of the radiation (accounting for the dip near the line center) was ignored so that theory and experiment may be compared for large detuning only, i.e. for $\left(\nu-\nu_{0}\right)$ considerably larger than $\gamma_{h}^{\prime}$. It will appear that this condition holds near threshold detuning in case of fig. 1 . It is seen that there is good agreement between the theoretical curve 2 and experiment, indicating that the laser power output is proportional to the square of the gain curve (cf. eq. (1)) rather than proportional to the first power (cf. eqs. (2) and (3)).

The underlying power broadening can be found directly as follows. The deviation between curves 1 and 2 around the line center is evidently due to the neglect of the standing-wave nature of the radiation. Thus from the ratio (curve 1)/(curve 2) we can obtain the shape of the function which describes the increased saturation around the line center due to the overlapping holes in the gain curve. A graphical analysis of curve 3 learns that it is a Lorentzian function of detuning, as expected [4], with a half-width at half maximum of $\gamma_{h}^{\prime}=$ $=57 \pm 1 \mathrm{Mc} / \mathrm{s}$. A similar procedure applied to a measurement at the lower excitation level of $\eta \approx 1.25$ yielded $\gamma_{\mathrm{h}}^{\prime}=47 \pm 5 \mathrm{Mc} / \mathrm{s}$ (the error being determined mainly by the occurrence of an asymmetry in the experimental curve). Thus we demonstrated directly the power broadening of the holes burnt in the gain curve.

We conclude that through our modulation measurements [6-9] one can separate experimentally

* The ratio $r$ does not contain power broadening in this case.

\# In fact, we calculated the theoretical curves for $r=$ $=0.1$ by means of the exact expression given by Smith. This magnitude of $r$ was approximately known from our earlier measurements $[6,8]$. 
between collision - and power broadening. In addition, it is clear that the determination of the spontaneous-emission lifetime and of collision broadening of the laser transition by means of the Lamb dip yields incorrect results, when the effect of the stimulated emission on the lifetime is not taken into account, in particular so at the lower gas pressures and the higher excitation levels $[1,14]$. Note further that the modulation method may be useful in proving the quadratic dependence of the laser power on the excitation level (cf. eq. (1)), which is also predicted by Willis' quantum theory of a gas laser [15].

More details about this work were given in ref. 13.

The authors wish to express their appreciation to the N.V. Philips' Gloeilampenfabrieken for supplying the laser.
1. A.Szöke and A.Javan, Phys. Rev. 145 (1966) 137.

2. P.W.Smith, J. Appl. Phys. 37 (1966) 2089.

3. W. E. Lamb Jr., Phys. Rev. 134 (1964) A 1429.

4. W. R. Bennett Jr., in: Proc. 3rd Int. Congr, on Quantum electronics, Paris, 1964, eds. P.Grivet et al. (Columbia University Press, New York, 1964) p. 441 .

5. K. Uehara and K.Shimoda, Japan J. Appl. Phys. 4 (1965) 921.

6. P.T.Bolwijn, Phys. Letters 19 (1965) 384

7. P.T.Bolwijn, IEEE J. Quant. Electron. QE2 (1966) 670.

8. P.T. Bolwijn, J. Appl. Phys. 37 (1966) 4487.

9. Th. H. Peek, P.T. Bolwijn and C.Th. J.Alkemade, Phys. Letters 24A (1967) 128.

10. P.W.Smith, IEEE J. Quant. Electron QE2 (1966) 62 .

11. I. Tobias, J. Appl. Phys. 34 (1963) 3200.

12. H.G. van Bueren, J. Haisman and H. de Lang, Phys. Letters 2 (1962) 340.

13. P.T.Bolwijn, Noise, modulation and Zeeman effects in He-Ne lasers, Thesis, (Utrecht, 1967).

14. M.A. Pollack, T.J.Bridges and A.R.Strnad, Appl. Phys. Letters 10 (1967) 182 .

\title{
ANGULAR DEPENDENCE OF THE COLLISION-INDUCED EXCITATION OF H
}

\author{
D. K. GIBSON, J. LOS and J.SCHOPMAN \\ F. O.M.-Instituut voor Atoom-en Moleculfysica, Amsterdam, The Netherlands
}

Received 21 September 1967

\begin{abstract}
Some measurements of the angular dependence of the collision-induced excitation cross section of $\mathrm{H}_{2}^{+}$are presented*. These measurements suggest that simultaneous excitation of the target atom is not negligible.
\end{abstract}

It is now commonly assumed that the collisioninduced dissociation of $\mathrm{H}_{2}^{+}$ions is mainly caused by the excitation of the $\mathrm{H}_{2}^{+}$ion from the ground state to an antibounding excited state, and the subsequent dissociation of the excited molecule. Dunn [1] has shown, using general symmetry arguments, that the excitation strongly depends on the angle between the intermolecular axis of the molecule and the direction of the momentum transfer vector $K$, provided the Born approximation is valid. For the excitation to the 2 pou state of $10 \mathrm{keV} \mathrm{H}+\mathrm{H}_{2}^{+}$ions incident on noble gas atoms, the average direction of the $K$ vector is approxi mately antiparallel with the incident beam direction for small internuclear distances $R$, because

\footnotetext{
* In the course of this experiment we became aware that at the University of Giessen a group under Prof. $\mathrm{H}$. Ewald was studying the same problem, following however a different approach.
}

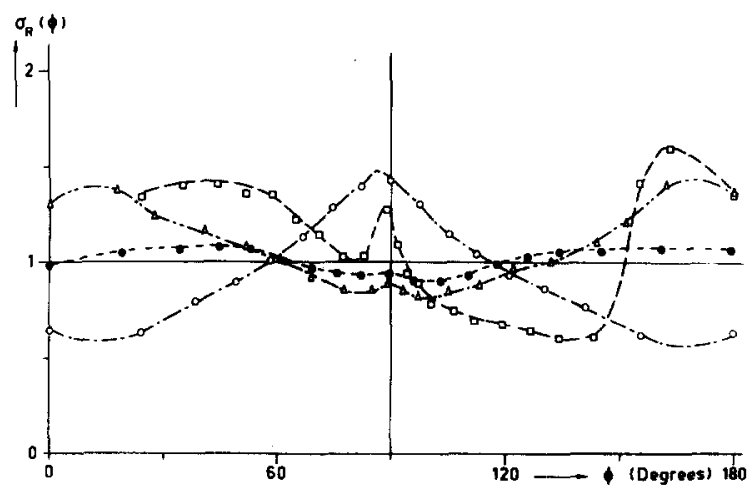

Fig. 1. Angular dependence of the dissociation cross section of $\mathrm{H}_{2}^{+}$incident at $10 \mathrm{keV}$ upon $\mathrm{He}$.

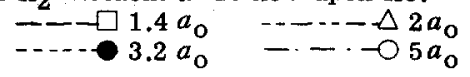

a large excitation energy is necessary. For large values of $R$, where the excitation energy is very 